\title{
Immobilization of CNFs on the surface and inside of the modified activated carbon
}

\author{
X.-W. Chen, D. S. Su*, and R. Schlögl \\ Department of Inorganic Chemistry, Fritz Haber Institute of the MPG, Faradayweg 4-6, 14195 Berlin, \\ Germany \\ * Corresponding author: e-mail: dangsheng@fhi-berlin.mpg.de, Phone: +49 308413 5406, Fax: +49 3084134401
}

The applications of carbon nanotubes or nanofibers (CNTs/CNFs) in chemistry and catalysis require the availability of large amount of materials with well-defined surface, chemical and mechanical properties. Loose CNTs/CNFs are unsuitable as they cannot be controlled in their suprastructural properties and operations of compaction can destroy or at least inhibit the access of the reactant to the nanostructures. It is mandatory for chemical applications to use only one chemical element for all dimensions of structuring and to avoid the combination of nanocarbon with noncarbon support structures. An ideal substrate is activated carbon (AC) from natural sources. Such bio-inorganic polymers are well structured in several dimensions, reactive for modifications and available in masses. The present work describes the ways to modify the activated carbon obtained from the bio-waste of palm oil production as host for the growth of nanocarbon. CNFs can be immobilized on the outer surface and nested inside of the activated carbon leading to hierarchically structured carbon materials that are highly suitable for sorption and catalytic applications and for binder-filler applications.

\begin{abstract}
1 Introduction
In chemical nanotechnology, CNTs/CNFs can be used for the selective chemisorption of unwanted species from drinking water (Mn, As, Hg, Fe), as additives for the modification of the properties of polymers and as catalysts or catalyst supports [1-7]. For instance, CNTs and CNFs exhibit excellent catalytic performance for oxidative dehydrogenation of ethyl benzene to styrene [8,9]. These applications require, however, the availability of large amount of materials with well-defined surface, chemical and mechanical properties. In addition, the nanocarbon must be formed into big particles to optimize the hydrodynamic properties allowing an effective contact with reacting matrices. Loose CNTs/CNFs are unsuitable because they cannot be controlled in their suprastructural properties and operations of compaction can destroy or inhibit the access of the reactant to the nanostructures. Pressure drop and hot spots are additional problems of loose CNTs/CNFs if they are used in the scale-up reactors. A hierarchical organization of the nanocarbon on carbon for small dimensions and on a robust carrier structure in larger dimensions is therefore highly desirable. It is mandatory for chemical applications to use only one chemical element for all dimensions of structuring and to avoid the combination of nanocarbon with non-carbon support structures as the resulting discontinuities in transport and in chemical properties will cause deterioration of the overall compound properties and cause chemical instability. This work describes the method to immobilize CNFs on modified activated carbon obtained from the bio-waste of palm oil production. Nanostructured carbon can be obtained on the outer surface of the activated carbon yielding a useful geometry for binder-filler applications. Alternatively, burning large pores into the bulk structure of the activated carbon and subsequent filling of the pores with carbon nanostructures leads to hierarchically structured carbon materials with nested CNFs inside.
\end{abstract}

\section{Experimental}

The preparation procedures were described in our previous paper [10]. The activated carbon used as support was supplied by COMBICAT research center, University of Malaya, Malaysia. It was made from palm kernel shell, a waste product from palm oil production. It contains besides carbon substantial amount of silicate and traces of iron as iron silicate after the activation. To use it as support, the activated carbon was calcined at $400{ }^{\circ} \mathrm{C}$ for $4 \mathrm{~h}$ in air. The $1 \mathrm{wt} \% \mathrm{Fe} / \mathrm{AC}$ precursor was prepared by impregnation using an aqueous solution of $\mathrm{Fe}\left(\mathrm{NO}_{3}\right)_{3}(0.09 \mathrm{M})$ and activated carbon calcined at $400{ }^{\circ} \mathrm{C}$ with an iron loading of $1 \mathrm{wt} \%$ in the catalyst. A drying step at $60{ }^{\circ} \mathrm{C}$ over night was followed by a calcination at $500{ }^{\circ} \mathrm{C}$ in $\mathrm{N}_{2}$ for 4 h. The Fe-impregnated sample was reduced with $\mathrm{H}_{2}$ at $700{ }^{\circ} \mathrm{C}$ for $4 \mathrm{~h}$. The $1 \mathrm{wt} \% \mathrm{Fe} / \mathrm{AC}$ catalyst (100 mg) was put into a vertical quartz reactor and flushed using He overnight. The carbon nanofibers were grown by treating the Fe/AC catalyst at $700{ }^{\circ} \mathrm{C}$ in flowing 60 vol\% $\mathrm{C}_{2} \mathrm{H}_{4} / \mathrm{H}_{2}(125 \mathrm{ml}$ $\mathrm{min}^{-1}$ ) at atmospheric pressure for $2 \mathrm{~h}$. The obtained sample was cooled down to room temperature in the flow of He. 


\section{Results and discussion}

A typical carbonised precursor obtained from palm kernel shell exhibits a BET surface area of $1081 \mathrm{~m} 2 \mathrm{~g}^{-1}$; the pore volume is $0.365 \mathrm{~cm}^{-1} \mathrm{~g}^{-1}$. To prepare the host for the designed hierarchical structure, the as-obtained AC was mildly oxidized at $400{ }^{\circ} \mathrm{C}$ in air for $4 \mathrm{~h}$. The activated carbon suffered a weight loss of about $5.2 \%$. Figure 1a shows a SEM image of activated carbon after the mild oxidation. Oxidation at this temperature removes the small debris of carbon from the surface and cleans the pores of activated carbon. Furthermore the oxidation enlarges the pore size.

The SEM image in Fig. 1b shows the catalyst of iron particles (Fe/AC) observed on the surface of the AC catalyst after the processes of impregnation, oxidation and reduction of catalyst. The specific BET surface area of Fe/AC catalyst is $1490 \mathrm{~m}^{-1} \mathrm{~g}^{-1}$ and the pore volume increases to $0.551 \mathrm{~cm}^{-1} \mathrm{~g}^{-1}$. After catalytic decomposition of a mixture of $\mathrm{C} 2 \mathrm{H} 4$ and $\mathrm{H} 2$ at $700{ }^{\circ} \mathrm{C}$ over the $\mathrm{Fe} / \mathrm{AC}$ catalyst, carbon nanofibers form and cover the activated carbon (as shown in Fig. 1c). They orient randomly and are entangled with each other. The diameters of the carbon nanofibers are widely distributed from 20 to $300 \mathrm{~nm}$. The SEM image confirms that the CNFs are grown on the outer surface of AC. In addition, the high magnification SEM study of individual CNF reveals the substantial surface roughness of the outer surface of the CNFs. Such a morphology is beneficial for the hydrodynamics of sorption processes from the liquid phase and provides mechanical and chemical anchoring sites when used as additive in polymer materials. The yield of the carbon nanofiber on a Fe/AC host reaches ca. $105 \mathrm{~g}$ of $\mathrm{C}$ per $1 \mathrm{~g}$ of Fe. The growth mechanism is the same as it is well known for chemical vapour decomposition (CVD) method to prepare CNTs/CNFs involving the decomposition, diffusion and precipitation of carbon atoms through the catalyst particle.

The high-resolution TEM image of the carbon nanofibers reveals that the carbon nanofibers are of the herringbone type disclosing the origin of the surface roughness. The graphite layers are declined to the fibers axis. With respect to the intended applications it was desired to expose as many prismatic faces as possible to the surface of the nanocarbon without losing all mechanical stability, making it more reactive as catalyst in heterogeneous catalysis.

Figure 1d presents a cross-section SEM image though the bulk of the CNFs/AC composite with two large pores in it after the CNF growth. Hair or micro bush structures are found inside the pore (comparing with the SEM image Fig. 1a). The high-magnification SEM study reveals that the dentritic structure is due to entangled CNFs created during the CVD process. The cross section TEM image of the pore proves convincingly that CNFs were grown on the inner wall of the pore. Moreover, the distribution of diameters of CNFs in the pores is quite narrow, in the range of 20-50 $\mathrm{nm}$. In addition, the CNFs grown inside the pores are shorter in length than these grown on the surface of the activated carbon. This could be due to a transport limitation of the ethylene into the pores.
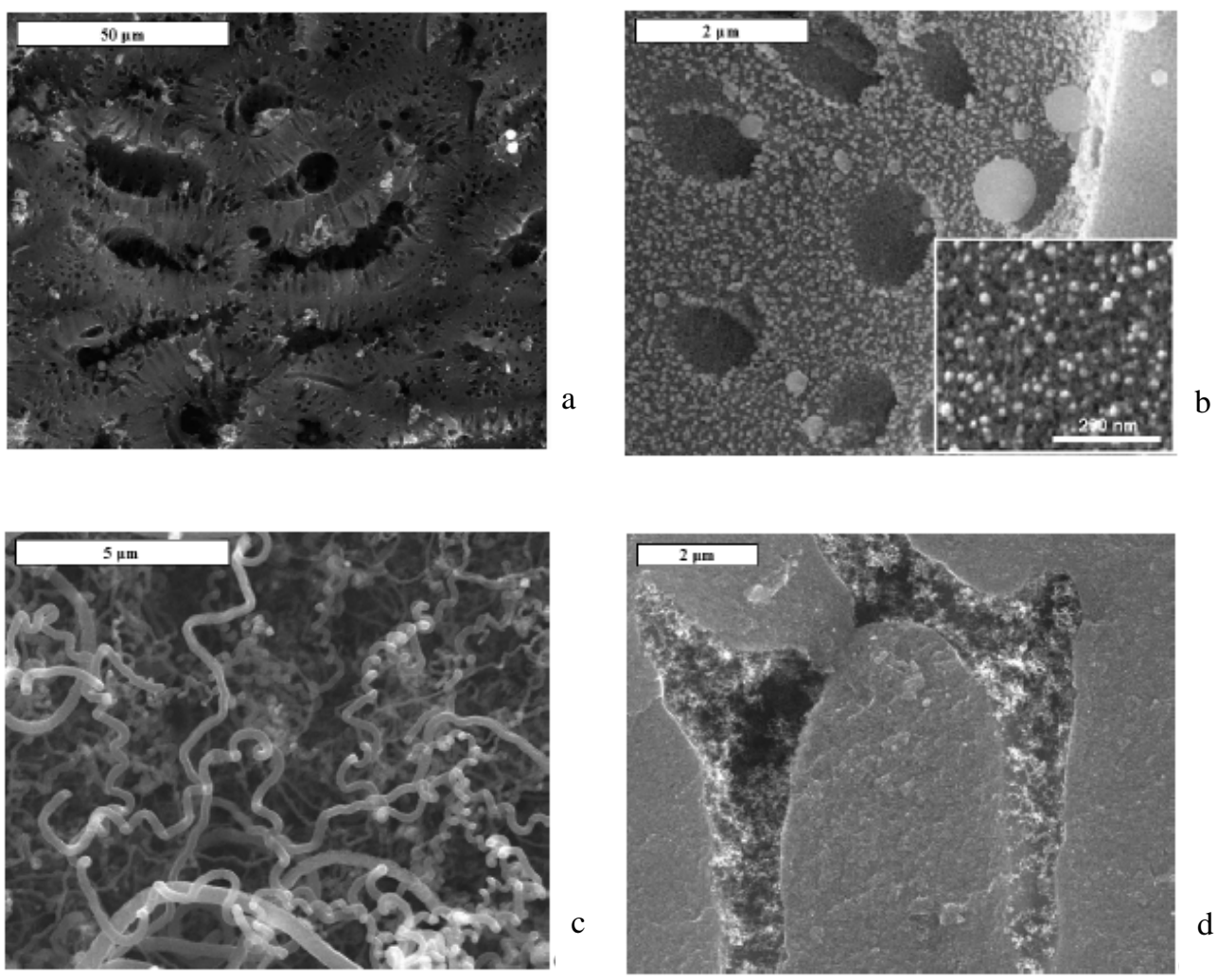

Fig. 1 a) SEM image of activated carbon calcined at $400{ }^{\circ} \mathrm{C}$, b) SEM image of $1 \mathrm{wt} \%$ Fe/activated-carbon, the inset is an enlarged image of Fe catalyst particles; c) SEM image of carbon nanofibers immobilized on the surface of activated carbon, d) cross-section SEM image of activated carbon showing carbon nanofibers grown inside the pore. 


\section{Summary}

In summary, hierarchically structured carbon with CNFs nested inside or immobilized onto the modified activated carbon are successfully synthesized by means of chemical vapour decomposition of ethylene. The activated carbon is obtained from bio-waste of palm oil production, followed by a mild oxidation as pre-treatment and the impregnation of catalyst. The method is scalable to mass production. The preliminary results of adsorption experiments serve as a proof of the concept that deliberate nanostructuring of a carbon surface can create novel functions of carbon that cannot be achieved alone by providing the adequate surface functional groups to the reactive medium. It is expected that the polymerisation process of metal-anionic species can be optimised by changing the pore size distribution, roughness of the CNFs and by adding to special surface functional groups.

Acknowledgements The work is partly supported by the Deutsche Forschungsgemeinschaft (Project SCHL 332), performed in frame of ELCASS, and partly supported by European Commission in the Sixth Framework Programme (NMP-500096, CANAPE).

\section{References}

[1] C. Pham-Huu, N. Keller, L. J. Charbonniere, R. Ziessel, and M. J. Ledoux, Chem. Commun. (19), 1871 (2000).

[2] H. X. Luo, Z. J. Shi, N. Q. Li, Z. N. Gu, and Q. K. Zhuang, Anal. Chem. 73, 915 (2001).

[3] R. Gao, C. D. Tan, and R. T. K. Baker, Catal. Today 65, 19 (2001).

[4] C. H. Liang, Z. L. Li, J. S. Qiu, and C. Li, J. Catal. 211, 278 (2002).

[5] T. G. Ros, A. J. van Dillen, J. W. Geus, and D. C. Koningsberger, Chem. Eur. J. 8, 2868 (2002).

[6] M. L. Toebes, F. F. Prinsloo, J. H. Bitter, A. J. van Dillen, and K. P. de Jong, J. Catal. 214, 78 (2003).

[7] V. Lordi, N. Yao, and J. Wei, Chem. Mater. 13, 733 (2001).

[8] G. Mestl, N. I. Maksimova, N. Keller, V.V. Roddatis, and R. Schlögl, Angew. Chem., Int. Ed. 40, 2066 (2001).

[9] D. S. Su, N. I. Maksimova, J. J. Delgado, N. Keller, G. Mestl, M. J. Ledoux, and R. Schlögl, Catal. Today 102/103, 110 (2005).

[10] D. S. Su, X. Chen, G. Weinberg, A. Klein-Hofmann, O. Timpe, S. B. A. Hamid, and R. Schlögl, Angew. Chem., Int. Ed. 44, 5488 (2005). 